\title{
Study of Optical Fibers Strain-Temperature Sensitivities Using Hybrid Brillouin-Rayleigh System
}

\author{
Kinzo KISHIDA*, Yoshiaki YAMAUCHI, and Artur GUZIK \\ Neubrex Co. Ltd., Sakaemachi-dori 1-1-24, Chuo-ku, Kobe, Hyogo 650-0023, Japan \\ *Corresponding author: Kinzo KISHIDA_Ｅ-mail: kishida@neubrex.jp
}

\begin{abstract}
In this paper, the most recent progress as well as challenges of distributed optical fiber sensing (DOFS) in industrial applications is discussed. Compared to the vast market of sensors used to measure strain or temperature, the success of distributed optical fiber sensing (DOFS) at the industrial level is very limited, at best. One of the reasons for this lack of the wider acceptance is the mismatch between the commercially available systems and actual industrial requirements, especially for the spatial resolution and precision. These requirements are organized and clarified in the paper. It also describes the hybrid Brillouin-Rayleigh system, which exhibits capabilities surpassing those of strain gauges. The principles of the system are illustrated considering the fiber calibration methodology. Formulas required for determining strain, temperature, and hydro-pressure are derived and discussed. Finally, the examples of applications are presented.
\end{abstract}

Keywords: Hybrid Brillouin/Rayleigh system, cross-sensitivity, material dependence

Citation: Kinzo KISHIDA, Yoshiaki YAMAUCHI, and Artur GUZIK, "Study of Optical Fibers Strain-Temperature Sensitivities Using Hybrid Brillouin-Rayleigh System," Photonic Sensors, 2014, 4(1): 1-11.

\section{Introduction}

The last 20 years saw an unprecedented progress in the development and implementation of fiber optics sensors. In general, fiber sensors fall into two categories of point-wise and distributed ones. The former, among others, includes the fiber Bragg gratings (FBG) and Fabry-Perot sensors. In essence, they can be considered as the optical counterparts of traditional electrical sensors, in which the implanted grating structure or reflective surface performs the sensing task, while the fiber itself is just a light guide and as such does not affect the sensor properties. For distributed sensors, the entire fiber takes a role of the sensor, and the glass properties influence its performance. The density fluctuations, the speed of acoustic wave, and the molecular free energy are the factors affecting the backscattering light, corresponding to Rayleigh, Brillouin, and Raman scatterings, respectively.

In Table $1, T, \varepsilon$, and $P$ stand for temperature, strain, and pressure, respectively.

Table 1 Status and trends in fiber sensing.

\begin{tabular}{c|c|c|l}
\hline Principle & $\begin{array}{c}\text { Sensing } \\
\text { target }\end{array}$ & $\begin{array}{c}\text { Suitable } \\
\text { fiber }\end{array}$ & \multicolumn{1}{c}{ Recent trend } \\
\hline Brillouin & $T, \varepsilon, P$ & $\mathrm{SM}$ & $\begin{array}{l}\text { S-BOTDR single-end cm } \\
\text { resolution } \\
\text { Acoustic sensing, phase } \\
\text { Rayleigh }\end{array}$ \\
$T, \varepsilon, P$ & $\mathrm{SM}$ & $\begin{array}{l}\text { analysis } \\
\text { Faster acquisition }\end{array}$ \\
\hline
\end{tabular}

In this paper, the most recent progress as well as challenges of distributed optical fiber sensing in industrial applications is discussed. The spatial 
resolution, precision, measurement speed, and cross sensitivity are described in detail.

\section{Requirements from mechanics perspective}

The strain levels encountered in engineering structures vary considerably. Their working ranges are determined at the design stage and usually limited to several hundred micro-strains. So far, in the wide range of industries including automobiles, aviation, and civil infrastructure, the strain is measured almost exclusively using strain gauges. Strain gauge courses are fundamental in any mechanical engineering curriculum, and every big manufacturer employs hundreds if not thousands qualified strain gauge engineers. In the light of this, the success of optical fiber sensing at the industrial level is, at its very best, very limited. One of the reasons for the lack of the wider approval is the miscommunication and sometimes failure to understand differences between challenges and requirements in optics and structural mechanics. This can be illustrated considering the mechanics principles of steel.

For the typical carbon steel, the yielding point value, that is, as the point at which a material starts to deform plastically is about 1000 micro-strains. An example of such strain-stress curve for steel S-45 C is presented in Fig. 1. Other selected examples of typical levels are listed in Table2.

For the design of every structure, a safety factor $n_{s}$ is used. For the structures like the bridge, this factor is from 5 to 8 . The lowest one is for a plant, which is 2 . Then, the maximum strain is limited, by the design of the strength, as $\varepsilon_{\max }<\varepsilon / n_{s}$, and the values are listed in Table2.

The optical fiber, to be considered as an actual sensor and competitors with the strain gauges, must be able to measure the strain changes, namely, detect changes at least one order in magnitude smaller than those listed in Table2. The actual request would be two orders $(1 / 100)$ smaller, i.e. several micro-strains.

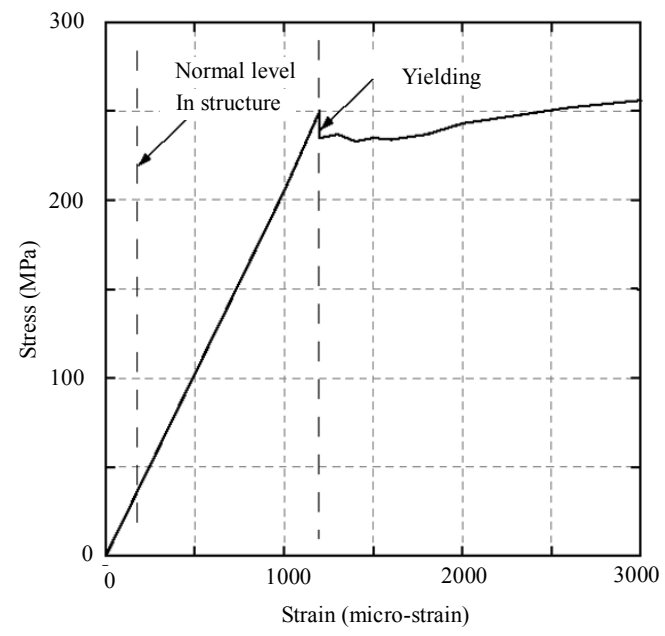

Fig. 1 Stress-strain curve for steel S-45C.

Table 2 Typical strain levels in specific applications.

\begin{tabular}{cc}
\hline Structure & Strain level $(\mu \varepsilon)$ \\
\hline Civil structure & $100 \sim 200$ \\
Chemical plant & $400 \sim 700$ \\
Concrete (before crack) & 5 (tensile) $100 \sim 200$ (compressive) \\
Steel-reinforced concrete & $<100$ up to above 20,000 at cracks \\
\hline The distributed & Sensing $\quad$ Systems, both
\end{tabular}
instruments and fiber cables, must meet several requirements to create for the user/customer an added-value and be economically justifiable. Among these requirements are precision, spatial resolution, distance compensation, and temperature-strain separation.

As the hybrid Brillouin-Rayleigh system combines Brillouin and Rayleigh backscattering phenomena, the requirements for each sub-system will be described separately, for the clarity of presentation.

\section{Hybrid Brillouin-Rayleigh system}

The Brillouin sensing systems, well-known and used, cannot meet the requirements described in the previous section and thus will not be the replacement for the strain gauges. The other well-known and used technology which can meet tho se is FBG.

In this section, we will describe the Brillouin system first and then present the time domain 
Rayleigh sensing, the tunable wavelength coherent optical time domain reflectometry (TW-COTDR). Next the hybrid system, which is expected to be an appropriate replacement for strain gauges, is described, while its applications are also presented.

\subsection{DTSS Brillouin-based sensing}

The Brillouin scattering is the backscattering of light by acoustic waves (phonons) in a medium and can be employed for distributed strain/temperature sensing. The position of the spectral peak varies linearly with the strain or temperature, that is, the value of the strain, $\varepsilon$, and/or temperature, $T$, can be estimated by measuring the shift of the spectral peak frequency. The linear dependency on the strain and temperature can be expressed as

$$
\Delta v_{B}=C_{11} \Delta \varepsilon+C_{12} \Delta T
$$

where $C_{11}$ and $C_{12}$ stand for the Brillouin strainfrequency and temperature-frequency coefficients, respectively.

The Brillouin scattering can be spontaneous or stimulated. The former is referred to as Brillouin time domain reflectometry (BOTDR), while the latter as Brillouin time domain analysis (BOTDA). An example of the Brillouin gain spectrum (BGS) of the BTODA is presented in Fig. 2.

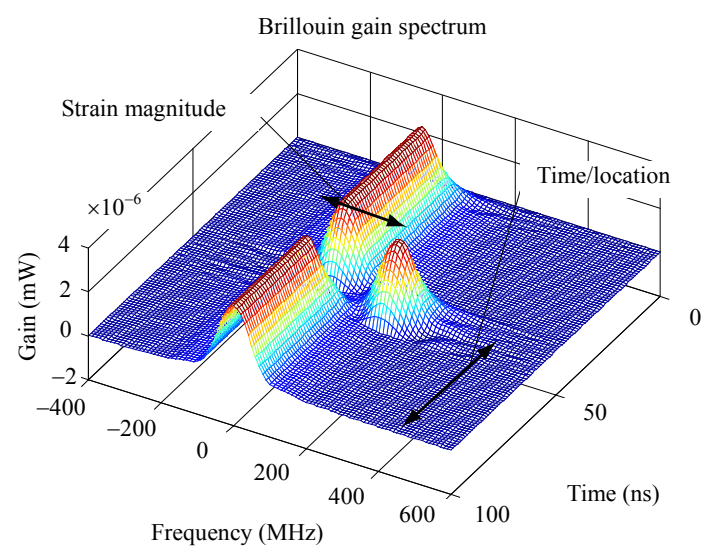

Fig. 2 Brillouin gain spectrum.

The time axis is equivalent to the distance as these two quantities are related by a multiplier, that is, the group velocity of light within the fiber. The shifted peak at about 50 ns (see Fig. 2) denotes the induced strain at that location. It should be noted here, however, that the shift and transition between the strained and unstrained portions are smooth, as the measured BGS is a convolution of the timevarying Lorentzian spectrum and the time-frequency window function.

Brillouin based sensing is by now the well established technology, providing fast and robust measurements with the reasonable resolution, commonly referred to as distributed temperature and strain systems (DTSS). The classical versions of the BOTDR and BOTDA are limited to the 1-m spatial resolution. The breakthrough technologies of the pulse-pre-pump (PPP) BOTDA [1] and especially synthetic-BOTDR [2] reduced the spatial resolutions to $2 \mathrm{~cm}$ and $10 \mathrm{~cm}$, respectively.

The duration of the pump pulse should be longer than the phonon relaxation time. Both experiments and simulations demonstrate that $28 \mathrm{~ns}$ is required to get the phonon fully stimulated. This corresponds to the measurement of the spatial resolution of about $3 \mathrm{~m}$. At this resolution, the full width at half maximum (FWHM) is approximately $33 \mathrm{MHz}$. The spectrum line width directly influences the precision of the central frequency estimation. Horiguchi [3] used exactly this parameter to illustrate the spatial resolution limitation of his invention at a very early stage. The error of the Brillouin measurement can be expressed as

$$
\sigma_{v_{B}}^{2} \sim C \frac{b \Delta v}{S N R}
$$

where $b$ is the peak width (FWHM) of the measured Brillouin spectrum, $\Delta v$ is the frequency step, $S N R$ is the signal-to-noise ratio, and $C$ is a constant depending only on the ratio $r=$ (width of frequency interval used for curve fitting) $/ b$. When $r$ is equal to one, $C$ takes the value of about 0.9 , and when $r$ tends to infinity, $C$ approaches to $2 / \pi$.

From the above equation, it is clear that increasing the spatial resolution decreases the measurement error. In fact, if the precision of measurements is determined by repeatability, as 
used by majority of the manufactures, most of commercially available instruments can reach the precision as small as 20 micro-strains. Unfortunately, this is not true in the actual applications.

An example of the actual measurement strain distribution is presented in Fig. 3. It clearly demonstrates the residual strain presented in every fiber.

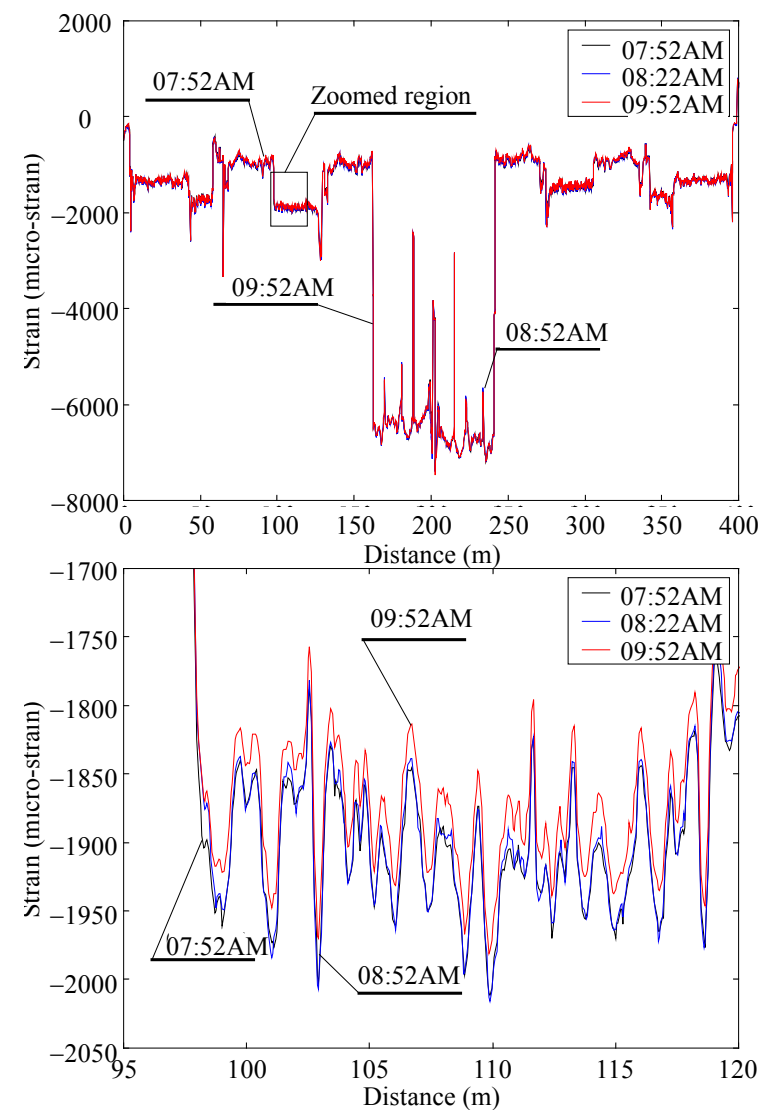

Fig. 3 Example of the residual strain distribution.

This is unknown to most of the users and well concealed by most of manufacturers. The ripples in the strain distribution have the period of approximately 1 meter, which matches well with the length of one turn of the optical fiber around the standard 26-cm-diameter reel used in the production and storage of the fibers. If the spatial resolution is too large to observe these ripples, it implies that the strain change in the monitored structure will be higher than the amplitude of the residual strain ripples, which is around 100 to 200 micro-strains.
The minimum spatial resolution, required for the observation and thus accounting for the residual strain, is $20 \mathrm{~cm}$. From the accuracy perspective, as the 20 micro-strains is equivalent to $1 \mathrm{MHz}$ of the Brillouin central frequency shift, the 200 micro-strains of the residual strain results in $10-\mathrm{MHz}$ broadening. This is the primary reason why many BOTDA instruments with several meters resolution have the BGS line width of just $40 \mathrm{MHz}$ but still cannot detect many events.

The line width of the PPP-BOTDA is adjustable by changing the cross-talk of the main and pre-pump pulses. The line width is adjusted to $60 \mathrm{MHz}-$ $80 \mathrm{MHz}$, which is doubled as that for the standard BOTDA with the 2-meter spatial resolution. However, our experience in the field shows that the error is smaller than 10 micro-strains if the proper distance compensation is implemented. The line width in S-BOTDR measurements is for any resolution smaller than $40 \mathrm{MHz}$. Based on this analysis, the precision limits for each Brillouin scattering based technology can be determined. The results are listed in Table3.

Table 3 Precision achieved by different technologies.

\begin{tabular}{ccl}
\hline Technology & Precision $(\mu \varepsilon)$ & Main reason limiting the precision \\
\hline BOTDR & $\sim 200$ & $\begin{array}{l}\text { Residual strain in optical fiber and wide } \\
\text { line width }\end{array}$ \\
BOTDA & $\sim 150$ & Residual strain in optical fiber \\
PPP-BOTDA & $<10$ & Line width of Brillouin \\
S-BOTDR & $<5$ & Signal to noise ratio \\
\hline
\end{tabular}

Table 3 alone does not highlight the essential problem of using distributed sensing in real-life engineering applications. It is only visible when contrasted with the values of the actual strain.

It is clear that most of the available technology and instruments are not quite adequate for the task of monitoring engineering applications as the 10 micro-strains accuracy and at least $20-\mathrm{cm}$ resolution are required. Moreover, usually strain/ temperature monitoring is just the first step in the structure health monitoring (SHM), providing the 
data for subsequent structural analysis, where deformation, stress or other quantities of interest are used. It should be noted here also that fiber sensing provides the magnitude of the strain tensor in the direction of the fiber. Mapping these values onto the structural model requires the high resolution and accuracy in order to produce reasonable results from the analysis [4].

Yet another challenging and extremely important but never addressed problem in engineering applications is the distance compensation. As the speed of light in the fiber changes with the temperature and/or strain, and the change in the strain varies the fiber length, the changes in the actual locations of the measurement point should be considered at the data processing level. Unfortunately for the users, this problem is neither clearly illustrated nor solved by most of the instrument providers. As a result, most of the users draw incorrect conclusions from their measurements and/or try to develop the necessary tools themselves.

In the DTSS systems, both temperature and strain changes produce the shift in the central frequency. The stimulated Brillouin scattering (SBS) based instruments thus cannot separate strain and temperature contributions from the measured total shift (the temperature change of $1{ }^{\circ} \mathrm{C}$ is equivalent to the strain change of 20 micro-strains). So far, the most common approach to separate the strain and temperature is to use the strain-free fiber. Unfortunately, in industrial applications, the strainfree fiber is never actually free from the strain. Several other methods were also proposed including the Raman (DTS) based temperature compensation, amplitude measurement method [8], or polarizationmaintaining (PM) fibers [9].

The actual separation is possible only if an additional, independent relation between strain, temperature and shift is available. The required relation can be provided by the frequency shift of time domain Rayleigh backscattering [5].

\subsection{DTSS sensing using TW-COTDR}

The optical frequency domain reflectometry (OFDR) presented in [6] was the first attempt to utilize the principle of Rayleigh backscattering in optical fibers for the strain measurement. Koyamada [7] proposed a different, time-domain approach, with important advantages of the long distance and high accuracy. As to this method, the random in-homogeneity of the glass density in the fiber core manifests itself as refractive index fluctuations, observed as the random distribution of the power spectrum in the coherent OTDR (C-OTDR) trace. It can be implemented by means of the tunable wavelength of distributed feedback (DFB) laser and frequency scanning to obtain the power spectrum (TW-COTDR). This is mathematically equivalent to the impulse response of the pump light pulse and the Gaussian white noise distribution, representing the above mentioned density (refractive index) fluctuations. Figure 4 presents the Rayleigh spectrum measured using the $10-\mathrm{cm}$ spatial resolution and single-mode (SM) fiber.

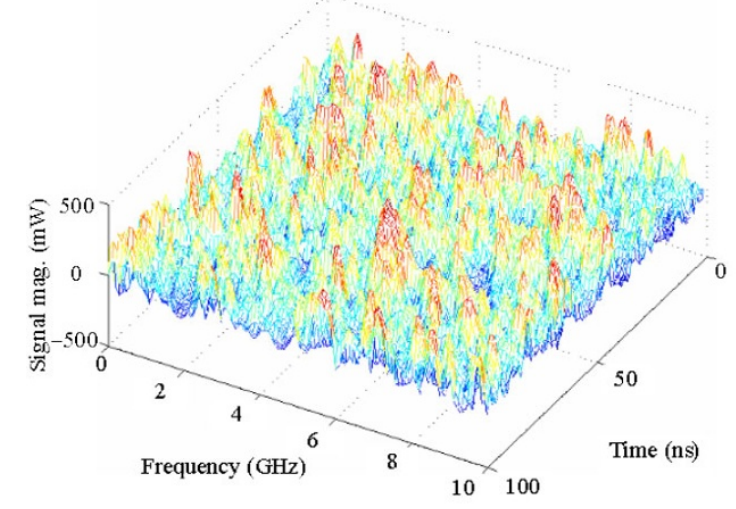

Fig. 4 Rayleigh scattering spectrum.

The measurements are compared with the reference state, and via cross-correlation, the frequency shift can be calculated. The frequency shift $\Delta v_{R}$ induced by the strain/temperature changes can be expressed using

$$
\Delta v_{R}=C_{21} \Delta \varepsilon+C_{22} \Delta T .
$$

The Rayleigh scattering signal is strong, offering thus high optical budget, and technically has the same sensitivity as the FBG, that is, is three orders 
of magnitude higher than that of the Brillouin scattering. Experiments during the development of the commercially available NBX-7020 instrument revealed that with the accuracy of the laser to be $12 \mathrm{MHz}$ the achievable precision for the strain is as high as 0.078 micro-strains or $0.01{ }^{\circ} \mathrm{C}$ for the temperature. Although the laser can be further improved, even the present level already surpasses the accuracy of strain gauges.

The only weakness of the TW-COTDR is related to the way the frequency shift is actually obtained. The non-uniform distribution of the strain and/or temperature within the spatial resolution leads to low correlation values (multi-peak distribution), and as a consequence, it is difficult to determine the value of the shift. Additionally, if the fiber undergoes the elongation larger than the spatial resolution, no correlation can be found, as the spectra at different locations are compared. As a result, the TW-COTDR requires an accompanying tool to be reliable in engineering applications. Fortunately, this tool is the Brillouin scattering.

\subsection{Hybrid Brillouin-Rayleigh sensing system}

The hybrid Brillouin-Rayleigh system is schematically presented in Fig. 5.

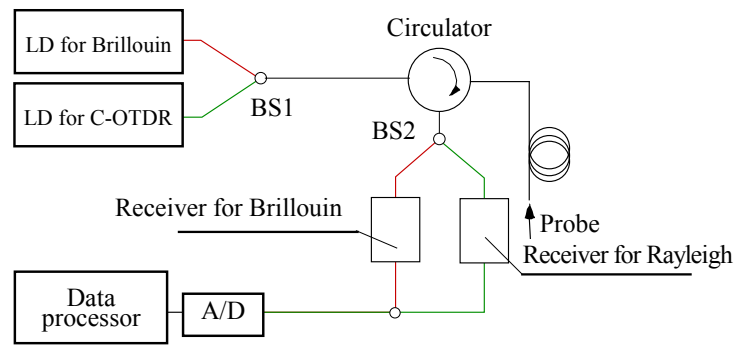

Fig. 5 Hybrid Brillouin-Rayleigh sensing system.

It consists of high-precision Brillouin (i.e. pulsepre-pump BOTDA), high-resolution tunable wavelength Rayleigh sub-systems, allowing one to measure Brillouin and Rayleigh scattering simultaneously.

The system provides for the SM fiber the measured shifts $\Delta v_{B}$ and $\Delta v_{R}$. By combining (1) and (3), the strain and temperature can be separated. This is of crucial importance in industrial applications.

The resolutions of the hybrid instrument vary from $2 \mathrm{~cm}$ to $1 \mathrm{~m}$, and the longest distance range covered in practice is $21 \mathrm{~km} \mathrm{[10].} \mathrm{The} \mathrm{combination}$ of Brillouin and Rayleigh sensing brings additional benefits in the form of improved data processing capabilities. Brillouin data are used for distance compensation and thus improve correlation analysis. In return, the Rayleigh frequency shift can be used to filter the Brillouin central frequency and improve the accuracy of the separated strain and temperature. This is schematically presented in Fig. 6, while Fig. 7 shows the filtered Brillouin central frequency distribution using the Rayleigh frequency shift.

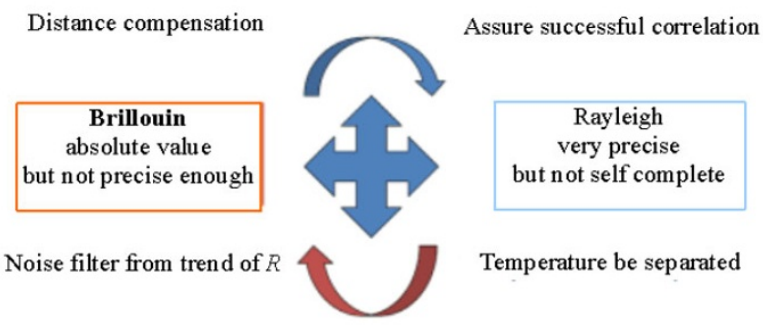

Fig. 6 Hybrid Brillouin-Rayleigh data analysis scheme.

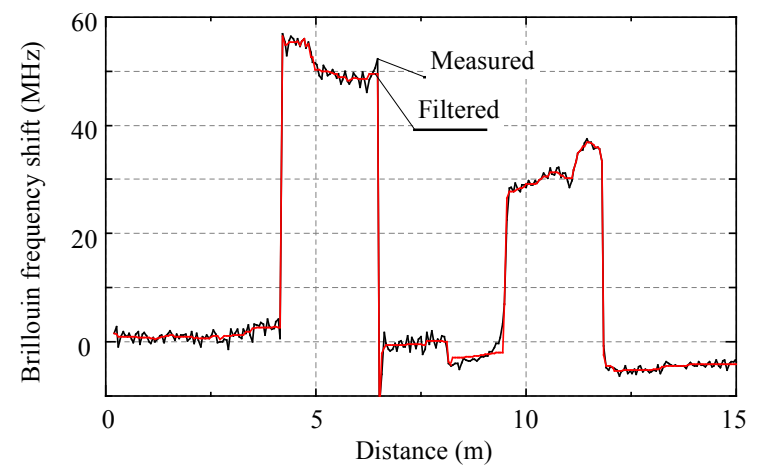

Fig. 7 Example of Brillouin central frequency filtered using the Rayleigh shift.

\section{Yamauchi's method for cross-sensitivity}

Engineering applications of the fiber optics usually require thickly coated fibers. The coating protects fibers from the mechanical damage, harsh environment and furthermore, provides means to attach, that is, install it, if used as a strain sensor. The long overlooked, or simply ignored problem, is the influence of thermal expansion of the coating on measurement results. Naturally, it can be generalized 
to fiber cables as well as cases when only the purely mechanical strain is required. Furthermore, external forces, including the pressure, as well as thermal expansion, and installation itself, induce the initial strain in the sensor. All these effects cannot be ignored and must be reflected in the strain and temperature cross-sensitivities.

In the remainder of this section, we describe in detail a simple yet extremely effective method for determining strain- and temperature-frequency coefficients and discuss the hysteresis effect in coated optical fibers.

\subsection{Yam auchi's method [11]}

This method is used to determine strain and temperature coefficients, and it relies on just the single basic assumption, namely, the attached fiber sensor exhibits the same thermal expansion as the underlying structure.

Based on these assumptions, the coefficients $C_{11}$ and $C_{22}$ can be split into two components:

$$
\begin{aligned}
& C_{12}=C_{12}^{\prime}+C_{11} \alpha \\
& C_{22}=C_{22}^{\prime}+C_{21} \alpha
\end{aligned}
$$

where $C_{12}^{\prime}$ and $C_{22}^{\prime}$ denote pure frequencytemperature coefficients for Brillouin and Rayleigh scattering, respectively, while $\alpha$ is the thermal expansion coefficient of the structure. Inserting (4) and (5) into (1) and (3) gives the following expressions:

$$
\begin{gathered}
\Delta v_{B}=C_{11} \Delta \varepsilon+\left(C_{12}^{\prime}+C_{11} \alpha\right) \Delta T \\
\Delta v_{R}=C_{21} \Delta \varepsilon+\left(C_{22}^{\prime}+C_{21} \alpha\right) \Delta T .
\end{gathered}
$$

For the strain free case, that is, $\Delta \varepsilon=0$ temperature measurements and two specimens (denoted in subsequent equations by superscripts $\mathrm{A}$ and B) of known thermal properties are required only. The system of equations for unknown coefficients takes the forms of

$$
\begin{aligned}
& \left\{\begin{array}{l}
\Delta v_{B}^{A}=\left(C_{12}^{\prime}+C_{11} \alpha^{A}\right) \Delta T \\
\Delta v_{B}^{B}=\left(C_{12}^{\prime}+C_{11} \alpha^{B}\right) \Delta T
\end{array}\right. \\
& \left\{\begin{array}{l}
\Delta v_{R}^{A}=\left(C_{22}^{\prime}+C_{21} \alpha^{A}\right) \Delta T \\
\Delta v_{R}^{B}=\left(C_{22}^{\prime}+C_{21} \alpha^{B}\right) \Delta T .
\end{array}\right.
\end{aligned}
$$

There is no restriction on selecting specimens. However, for the improved accuracy, they should exhibit distinctly different thermal expansion coefficients.

The practical implementation of this method uses only a temperature controlled heating bath and two cylinders and fiber wrapped spirally at the external surfaces of the cylinders, as shown in Fig. 8 .

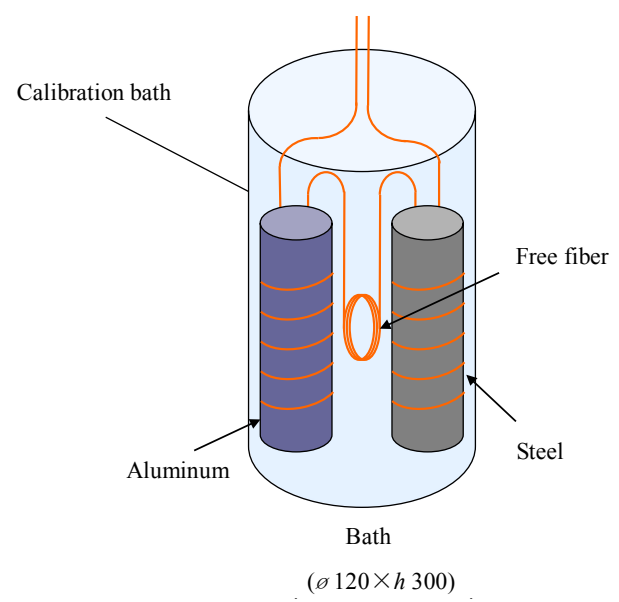

Fig. 8 Calibration bath and fiber arrangement.

Example results of the measurements for the $60-{ }^{\circ} \mathrm{C}$ temperature range (using $5{ }^{\circ} \mathrm{C}$ steps) are presented below.

The following equipment was used: calibration bath Julabo FK 31-SE, with the precision $\pm 0.005^{\circ} \mathrm{C}$ and temperature range from $-30{ }^{\circ} \mathrm{C}$ to $300{ }^{\circ} \mathrm{C}$; two $38 \mathrm{~mm}$ (diameter) $\times 250 \mathrm{~mm}$ (height) cylinders. Cylinders were made from steel and aluminum, with thermal expansion coefficients for steel and aluminum as high as 13.3 and 23.8 micro-strains $/{ }^{\circ} \mathrm{C}$, respectively.

The selected measurement results are presented in the following figures. Figures 9, 10, 11, 12, and 13 present measured temperature profiles, Brillouin central frequency, and Rayleigh frequency shifts.

Using that pre-processed measurement data, the gradients can be calculated. Solving systems (8) and (9) for unknown coefficients produces $C_{11}=$ $0.04904 \mathrm{MHz} / \mu \varepsilon, C_{12}^{\prime}=1.081 \mathrm{MHz} /{ }^{\circ} \mathrm{C}$, and $C_{21}=$ $-0.1542 \mathrm{GHz} / \mu \varepsilon, C_{22}^{\prime}=-1.379 \mathrm{GHz} /{ }^{\circ} \mathrm{C}$. 


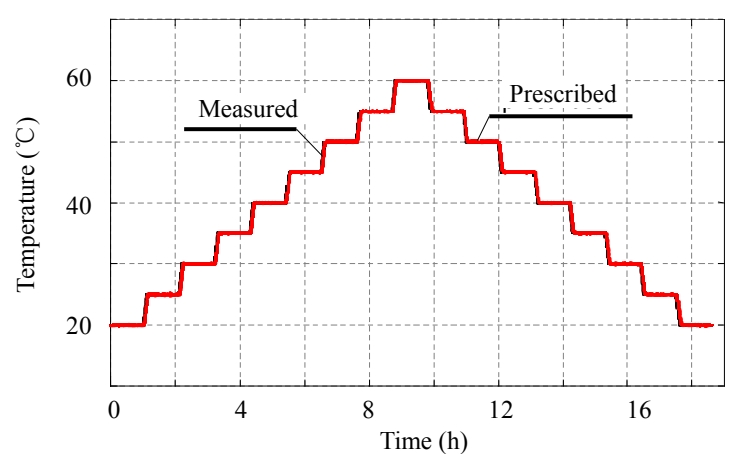

Fig. 9 Applied and measured temperature profile.

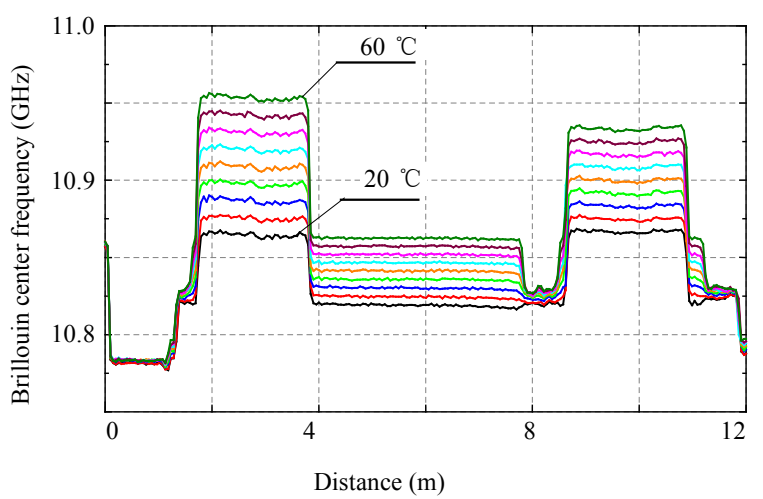

Fig. 10 Measured Brillouin central frequency.

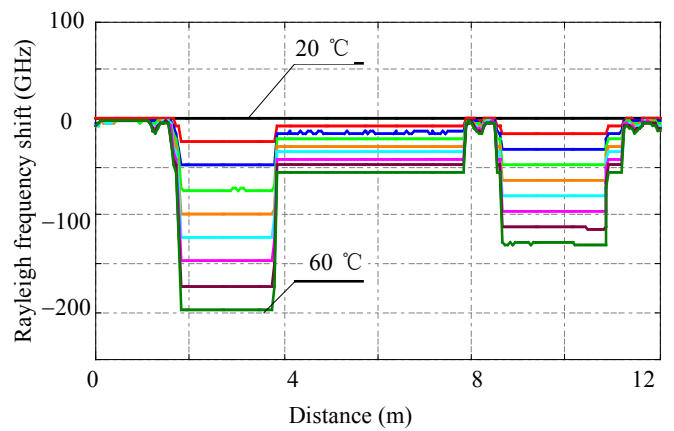

Fig. 11 Measured Rayleigh frequency shift.

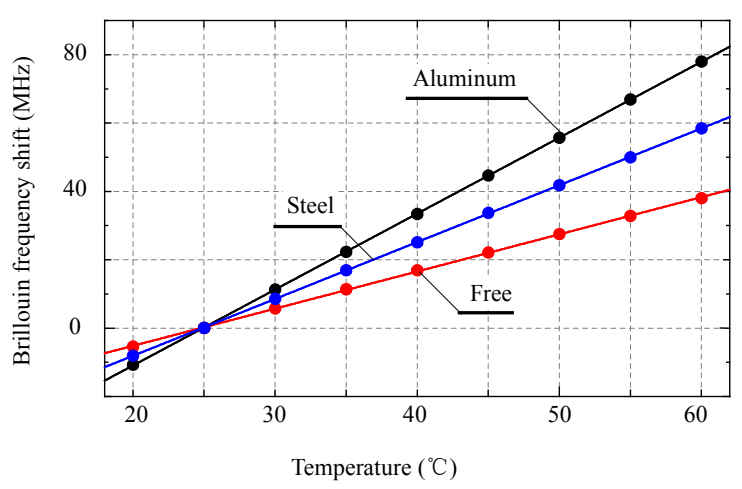

Fig. 12 Brillouin central frequency as a function of temperature.

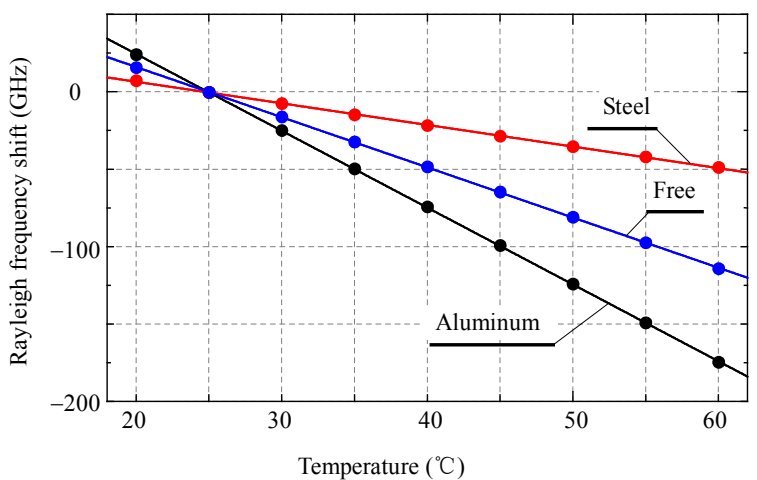

Fig. 13 Rayleigh frequency shift as a function of temperature.

\section{$4.2 R$ and $S$ parameters to determine temperature}

The fiber calibration method described above can still be improved by transforming (8) and (9) to get

$$
S=\frac{C_{21}}{C_{11}}=\frac{\Delta v_{R}^{A}-\Delta v_{R}^{B}}{\Delta v_{B}^{A}-\Delta v_{B}^{B}}
$$

and

$$
R=C_{22}-S C_{12}=C_{22}^{\prime}-S C_{12}^{\prime} .
$$

The practical implications of this transformation are far reaching. The calibration not only is much easier to perform but also produces more accurate results. Moreover, the coefficient $S$ is constant for almost all types of the fiber and equals -3091. An example of frequency differences in (10) is presented in Fig. 14.

Systems (6) and (7) can be expressed by using introduced coefficients $S$ and $R$ to give the equation independent of the strain:

$$
R \Delta T=\Delta v_{R}-S \Delta v_{B} .
$$

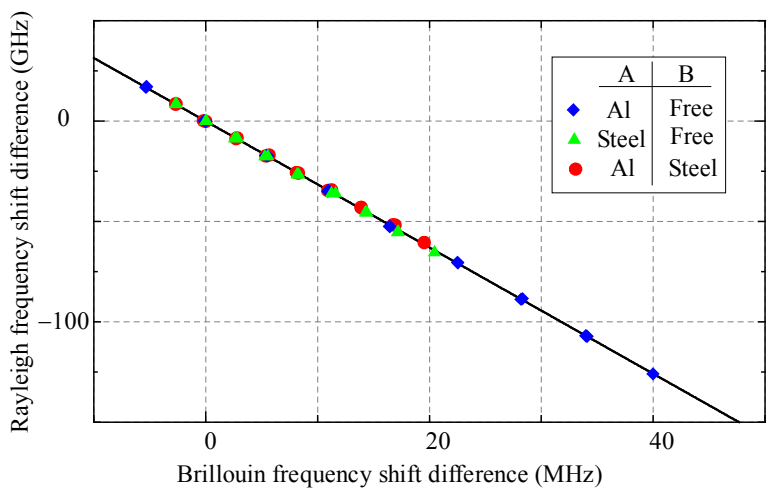

Fig. 14 Frequency differences in (10). 
Figure 15 shows that $R$ is a constant value $\left(2.289 \mathrm{GHz} /{ }^{\circ} \mathrm{C}\right.$ here), and the results presented in Fig. 16 are effective up to $150{ }^{\circ} \mathrm{C}$.

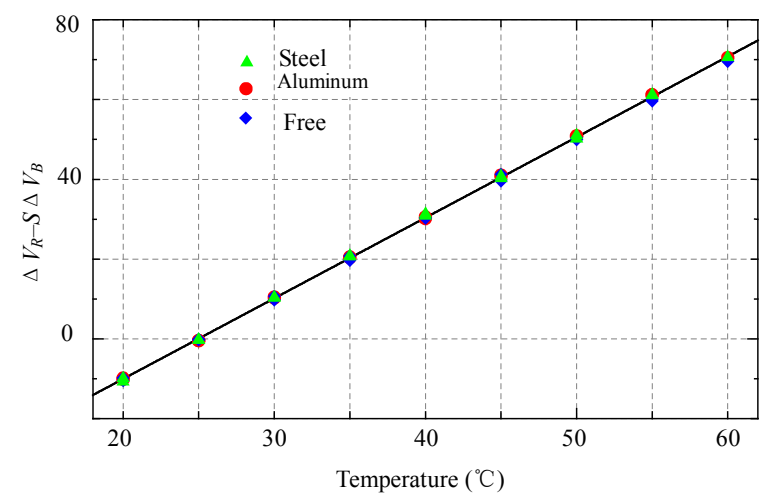

Fig. 15 Right hand side of (12) as a function of the temperature.

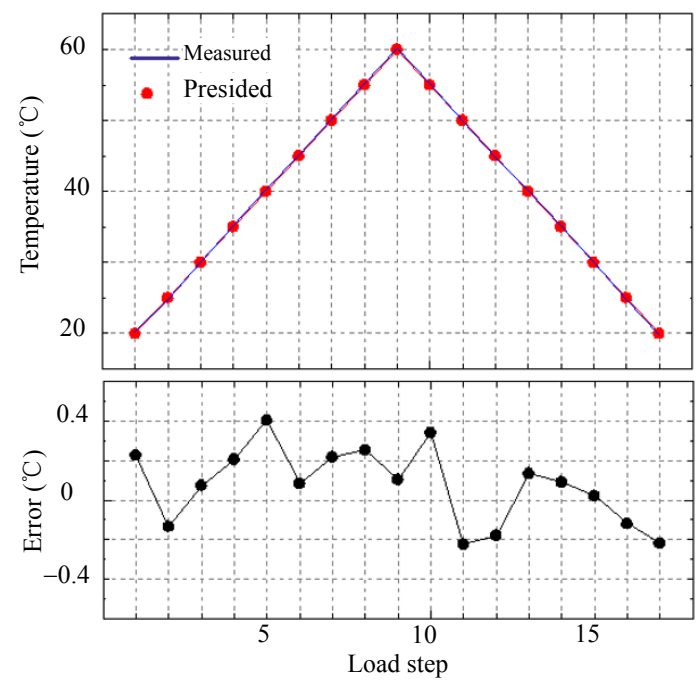

Fig. 16 Comparison of measured and prescribed temperatures using calibrated fiber coefficients.

Up to this point, the methods to measure cross sensitivities and obtain the correct temperature value independent of the measurement target were illustrated. Taking this consideration to the next step, that is, the determination of the mechanical strain, (6) and (7) lead to the following expressions for the mechanical strain

$$
\Delta \varepsilon=\frac{1}{C_{21}}\left(\Delta v_{B}-C_{22} \Delta T\right)
$$

and total strain

$$
\Delta \varepsilon_{\alpha}=\frac{1}{C_{21}}\left(\Delta v_{R}-C_{22}^{\prime} \Delta T\right)=\Delta \varepsilon+\alpha \Delta T .
$$

Naturally, for calculating the total strain, the value of the thermal expansion coefficient must be known. It should be noted here that this is also valid for strain gauges, and also for them, special steps are taken into account for the thermal deformation.

\subsection{Hysteresis effect}

The hysteresis effect of the coated and installed fibers is evident considering the warming up and cooling down process. Figures 17 and 18 present the results of frequency shift measurements during the first cycle of that process. It clearly demonstrates the substantial difference in obtained shift values.

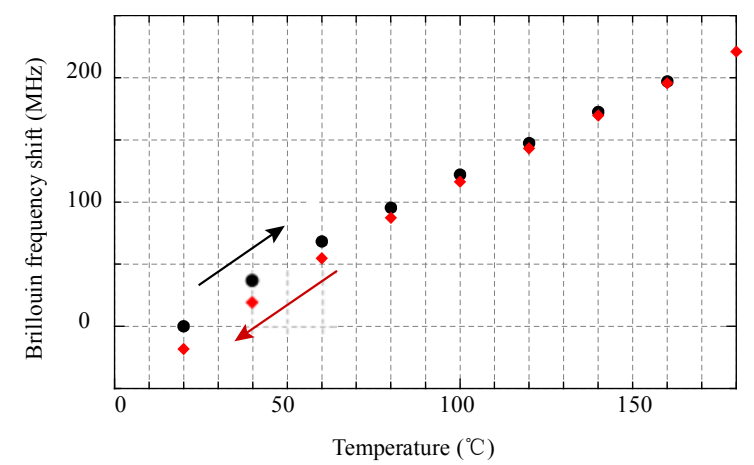

Fig. 17 Hysteresis effect of the optical fiber - Brillouin central frequency.

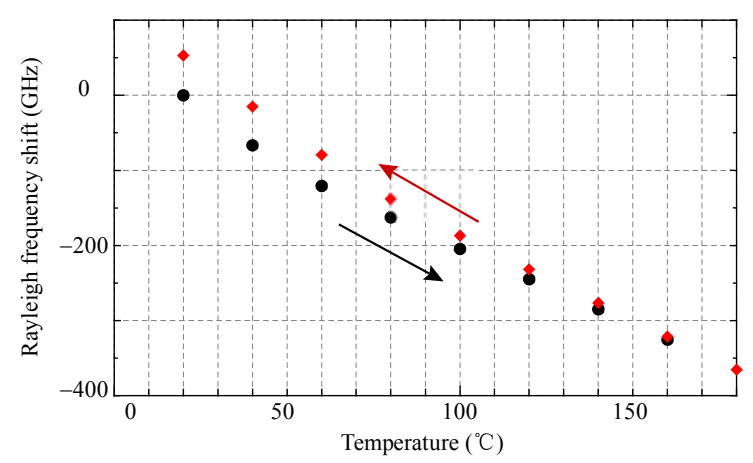

Fig. 18 Hysteresis effect of the optical fiber - Rayleigh frequency shift.

The effect disappears if a few cycles of heating and cooling are completed. The effect was detected and known to many engineers and fiber optics practitioners but the phenomena and reasons were never explained before. The updated temperature, calculated using parameters $R$ and $S$, is shown in Fig. 19. 


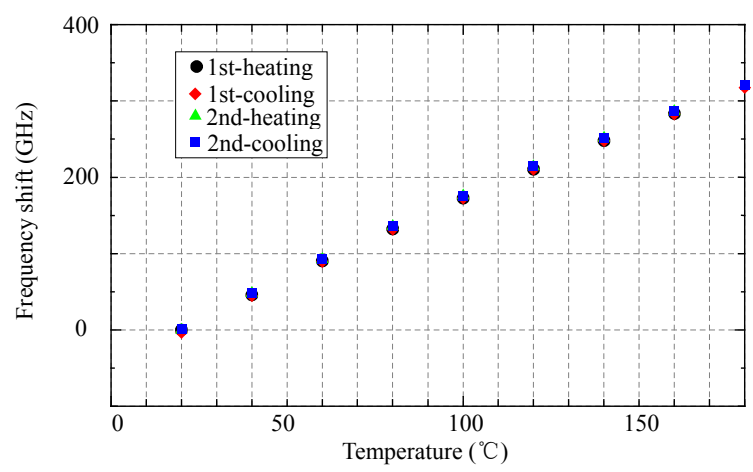

Fig. 19 Removal of the hysteresis effect using $S$ and $R$ parameters.

This time there is no frequency shift differences, and thus the influence is removed. All the tests demonstrated the same performance. The first cycle can be attributed to the strain variation caused by the adhesive material as well as the deformation of the coating materials.

\section{Optical fiber behavior under hydro- pressure}

One of the novel applications of the hybrid Brillouin-Rayleigh system is the measurement of the pressure distribution, which is of high interest in the fields such as oil, gas and geomechanics. The frequency shifts are linearly dependent on the pressure change up to $100 \mathrm{MPa}$ and can be expressed as

$$
\begin{aligned}
& \Delta v_{B}=C_{11} \Delta \varepsilon+C_{12} \Delta T+C_{13} \Delta P \\
& \Delta v_{R}=C_{21} \Delta \varepsilon+C_{22} \Delta T+C_{23} \Delta P .
\end{aligned}
$$

Figure 20 shows the frequency shifts as a function of the pressure at the room temperature.

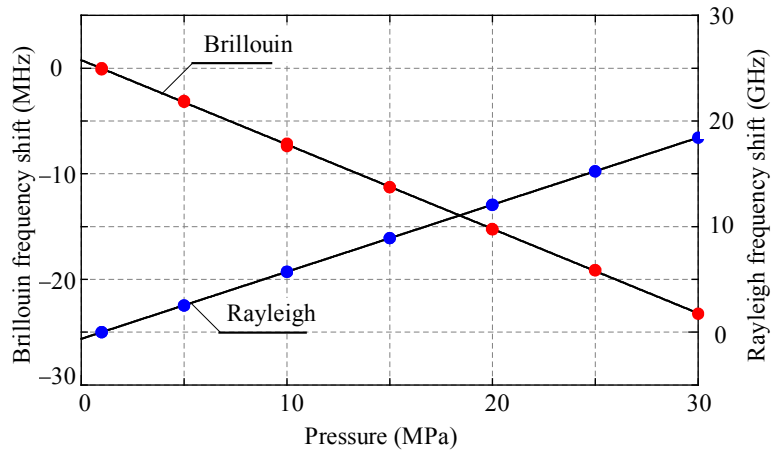

Fig. 20 Brillouin and Rayleigh frequency shifts as a function of pressure.
Some industrial reports suggested that the thicker coat should be used to increase the fiber sensitivity to the pressure change. Naturally, an increase in the external surface exposed to the pressure brings the desired effect on measured shifts, as demonstrated in Fig. 21.

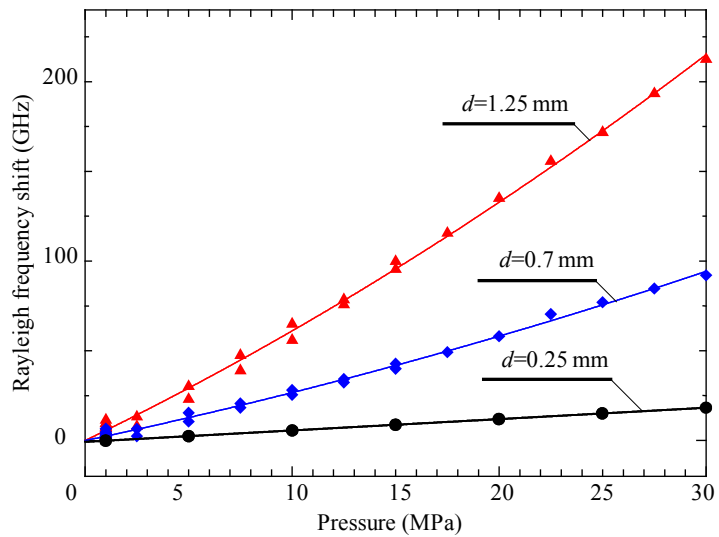

Fig. 21 Influence of the coated fiber diameter on the measured frequency shift due to the applied pressure.

Unfortunately, this behavior cannot be exploited in any practical application, especially in oil and gas, as it would require that both strain and temperature remain constant.

The hybrid system separates the influence of the pressure, temperature, and induced strain, and cancels this effect (of the increasing diameter of the coat).

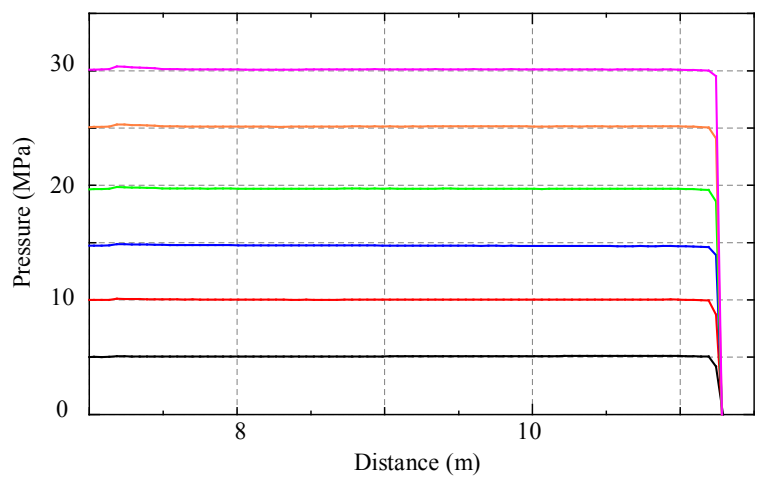

Fig. 22 Distributed pressure measurement.

Figure 22 presents example results of measurements performed by our group after eliminating the strain and temperature changes. The flat portion clearly demonstrates that the cable inside the hydro-pressure chamber gives the correct and 
stable pressure value. The error is below 3 psi $(20.7 \mathrm{kPa})$.

\section{Conclusions}

In the paper, the review of the requirements for distributed fiber sensing systems in industrial applications is presented. The spatial resolution, precision, and cross sensitivity are discussed in detail. Moreover, the principles of the hybrid Brillouin-Rayleigh system are outlined. Its implementation for calibrating the coated fiber parameters is illustrated. Finally, the paper presents the novel application of the hybrid system for measurements of the distributed pressure. Distributed optical fiber sensing is continuously evolving, finding new applications and new challenges.

\section{Acknowledgment}

We thank Prof. K. Nishiguchi, Osaka University, Japan, and Mr. C-H Li, Neubrex, for the valuable discussions.

Portion of the results presented in this paper was obtained during joint project with Mitsubishi Corporation, with financial support of Japan Oil, Gas and Metals National Corporation (JOGMEC). This support is gratefully acknowledged.

Open Access This article is distributed under the terms of the Creative Commons Attribution License which permits any use, distribution, and reproduction in any medium, provided the original author(s) and source are credited.

\section{References}

[1] K. Kishida, C. H. Li, and K. Nishiguchi, "Pulsed pre-pump method to achieve cm-order spatial resolution in Brillouin distributed measuring technique," Technical Report IEICE, 2004, 104(341): 15-20 (in Japanese).
[2] K. Nishiguchi, K. Kishida, and C. H. Li, "Synthetic approach for Brillouin optical time-domain Reflectometry," in The 42nd ISCIE International Symposium on Stochastic Systems Theory and Applications (SSS'10), pp. 81-88, 2010.

[3] T. Horiguchi, K. Shimizu, T. Kuroshima, M. Tateda, and Y. Koyamada, "Development of a distributed sensing technique using Brillouin scattering," Journal of Lightwave Technology, 1995, 13(7): 1296-1302.

[4] Y. Yamauchi, A. Guzik, K. Kishida, and C. H. Li, “A study on the stability, reliability, and accuracy of Neubrescope-based pipe thinning detection system," presented at The 3rd International Conference on Structural Health Monitoring of Intelligent Infrastructure (SHMII-3), Vancouver, Canada, Nov. 14-16, 2007.

[5] K. Kishida, C. H. Li, K. Nishiguchi, Y. Yamauchi, A, Guzik, and T. Tsuda, "Hybrid Brillouin-Rayleigh distributed sensing system," in Proc. SPIE, vol. 8421, pp. 84212G, 2012.

[6] M. Froggatt and J. Moore, "High-spatial resolution distributed strain measurements in optical fiber with Rayleigh scatter," Applied Optics, 1998, 37(10): $1735-1740$.

[7] Y. Koyamada, "New technique for distributed strain measurement in optical fibers with very high sensitivity by making use of Rayleigh backscattering", Technical Report of IEICE, OFT98-23, pp. 21-25, 1998 (in Japanese).

[8] T. R. Parker, M. Farhadiroushan, R. Feced, V. A. Handerek, and A. J. Rogers, "Simultaneous distributed measurement of strain and temperature from noise-initiated Brillouin scattering in optical fibers," Journal of Quantum Electronics, 1998, 34(4): 645-659.

[9] W. Zou, Z. He, and H. Hotate, "Complete discrimination of strain and temperature using Brillouin frequency shift and birefringence in a polarization-maintaining fiber," Optics Express, 2009, 17(3): 1248-1255.

[10] S. Delepine-Lesoille, A. Guzik, J. Bertrand, J. M. Henault, and K. Kishida, "Validation of TW-COTDR method for $25 \mathrm{~km}$ distributed optical fiber sensing," in European Workshop on Optical Fiber Sensors, 5th EWOFS'2013, Krakow, Poland, May 19-22, vol. 8794, 2013.

[11] Y. Yamauchi, "A measurement method to determine strain and temperature coefficients in fiber optic sensors," presented at Proc. of APWSHM Conference, Tokyo, Japan, 2010. 